\title{
COVID-19: Role of Deep Learning and Cloud Through Identification of Kidney, Pancreas and Intestine
}

\author{
Arulmozhiselvan $\mathrm{L}^{\mathrm{a}, 1}, \mathrm{Uma} \mathrm{E}^{\mathrm{a}}$ and Jayasri $\mathrm{R}^{\mathrm{a}}$ \\ a Department of Information, Science and Technology, Anna University, Chennai, \\ India
}

\begin{abstract}
Worldwide human health and economic has been affected due to the ongoing pandemic of corona virus (COVID-19). The major COVID-19 challenges are prevention, monitoring and FDA approved vaccines. IOT and cloud computing play vital role in epidemic prevention and blocking COVID-19 transmission. Mostly lungs and hearts are affected. Other than lungs many parts are affected which are not considered as prominent conversational cue. In this paper, we have proposed smart system that is effective through detection of pancreas, kidney and intestine. It detects acute pancreatitis, protein leak, microscopic blood leak, post infectious dysmotility and gastrointestinal bleeding. The data from the edge devices are collected and mapped into the cloud layer. The cloud consists of COVID-19 patients medical records which compare the user data with the existing patient records. Once the data matches it sends warning message to the user regarding the result of affected parts. Based on the result from KPI system, it analyzes with all data and using deep Convolutional Neural Network (CNN) it classifies whether the pancreas, kidney and intestine are affected or not due to COVID-19.
\end{abstract}

Keywords. Cloud Computing, KPI, Convolutional Neural Network, Acute Pancreatitis, Dysmotility.

\section{Introduction}

The COVID-19 is an ongoing pandemic of coronavirus disease 2019 caused by the transmission of severe acute respiratory syndrome coronavirus 2 (SARS-CoV-2), first identified in December 2019 in Wuhan, china. More than 43.9 million cases were recorded with more than 1.16 million deaths. It easily spreads through the respiratory route of infected person when they are physically close and spread through airborne. Though fever and cough are common symptoms; fatigue, difficulty in breathing and loss of smell and taste are other symptoms to identify the corona virus infection. Due to COVID-19 many complications may arises which includes pneumonia and respiratory problem. The life period of corona virus is around five days for moderate cases but ranges from 1 to 14 days. The global death-to-case ratio is 2.7 percent till October which is increasing day-by-day.

\footnotetext{
${ }^{1}$ L Arulmozhiselvan, Department of Information Science and Technology, Anna University, chennai.

E-mail: arulmozhiselvan@ auist.net.
} 
The metric used to assess the death rate is Infection Fatality Rate (IFR) which is calculated by taking deaths attribute to disease divided by infected individuals till date. COVID-19 spreads easily from one person to another through the respiratory path of an infected person while coughing, sneezing or breathing. Infected people remain in incubation period for 7-12 days in case of moderate and for severe cases it takes up to two weeks. In October 2020, it is clear that it can reinfect the patient with the same or different symptoms which were proved by scientists since the symptoms of COVID-19 are non-specific. Fever and dry cough are the common symptoms of corona virus.

Based on symptoms, COVID-19 can be diagnosed and confirmed using reverse polymerase chain reaction (RT-PCR) by taking the infected region or chest X-ray. Vaccine for corona virus is based on the knowledge about the structure and function which leads to development of vaccine in early 2020 through various technologies around the world. There were 321 candidates who developed vaccine to cure and prevent the virus infection, but the vaccines are under trail and testing regarding safety and efficiency. Other than lungs many organs are affected due to COVID-19. The organs such as kidney, pancreas and intestine are affected due to COVID-19. A research report shows that there is serious risk of Acute Kidney Injury (AKI) for people affected by COVID-19. It is the artifact to COVID-19 infection because it may lead to serious illness, dialysis and death rate is high for these patients. Various COVID-19 related effects lead to AKI which result in infection of the kidney. Even after discharged from hospital most of the COVID-19 patients have low kidney function.

COVID-19 cause acute pancreatitis. It may cause organ paralysis, failure of respiratory organ with high impermanence. Patients admitted with COVID-19 symptoms have upper abdominal pain. On analysis they had mild cough and unfiltered in chest X-ray on the day 3 of illness but no fever. There was no history of intake of alcohol, trauma or drug predisposing to AP. The patients were diagnosed as moderate COVID-19 and mild acute pancreatitis.

The Corona virus not only affects the lungs: in advanced cases of COVID-19 it causes gastrointestinal infection. Imaging scan reports of hospitalized COVID-19 patients reported with abnormalities in bowel region. Most of the effects were severe and leads to clots and blood flow impairment. Autopsy and biopsy are used to verify the intestinal damage caused by corona virus infection. 3\% of COVID-19 patients have gastrointestinal infection without respiratory infection.

A machine learning method has the ability to determine whether the COVID-19 patients will recover from complications or to be admitted in hospital based on the available data. The large dataset helped to ensure that the machine learning tool was able to read many different images and data available to predict the complications occurred due to COVID-19. It has also achieved in predicting hospitalization, illness and death. Machine learning tool potentially helps the providers and users to determine automatically and rapidly which patients are to develop complications from the virus infection.

The API machine learning tool helps to determine the acute pancreatitis, protein leak, microscopic blood leak, post infectious dysmotility and gastrointestinal bleeding based on the test report. It determines whether the patient organ is affected or not and store the data in the cloud. It helps to analyze the various patients and give advance alert rate about the complications. 


\section{Related Work}

The structure, diagnosis and vaccine of COVID-19 are discussed. The importance of deep learning, AI, IoT and blockchain in managing the severe effect of corona virus like false reports and wrong information [1]. Due to COVID-19 many organs are affected which are not in conversational cue. One of the organs is Kidney, a new automated sensing technique is introduced to detect the kidney disease by monitoring the urea level in saliva samples. And for further analysis of signals generated by sensor, the $\mathrm{CNN}$ is induced with SVM to classify [2].

Chronic Kidney Disease (CKD) has high death rate and causes other disease. In early stage, there are no symptoms to notice so the patients are not aware of disease. Corona patients are affected by chronic kidney disease which leads injury and function becomes slow. The unified model with perceptron; compared to other models it achieves high accuracy due to combination both logistic regression and random forest is proposed [3]. Corona virus causes pneumonia lesions and segmentation of lesions from CT images is important for accurate diagnosis [4]. Deep learning methods have the ability to automate the diagnosis and detection of disease which requires high quality data. The noise-robust framework called COPLE-Net helps to segment the lesions from the images automatically [5].

Wireless Capsule Endoscope (WCE) is used to detect the lesions in small intestine incorporated with YOLOv3 is proposed [6]. By comparing the hash values, the superfluous images are filtered. This computer-aided application with WCE detects the lesions accurately than manual detection. CKD results in high death rate, therefore it is necessary to detect the disease during early stage. Varieties of DL algorithm are proposed to classify the disease automatically [7]. But there is no work in comparing the DL algorithms which achieves more accuracy in CKD. The major issues of COVID-19 which includes geographical issues, high-risk patient and recognition have been reviewed [8]. For detecting and finding location of lesions in the kidney, the CT scan image plays a major role as it provides useful information in many applications. Traditional detection of lesions in kidney needs more labor and time. The proposed IOU based kidney lesion detection is based on cascade CNN to improve the tiny lesions and stability [9]. Other than CNN, FPN and RCNN are used in CT.

The diagnostic models for corona virus with multiple feature decision-making are not easy process. The TOPSIS is incorporated in MCDM method in order to standard the technique is to calculate weight of the multiple feature [10]. By analyzing various machine learning techniques, computer vision-based diagnosis of chronic Kidney Disease is proposed [11] and comparisons are made. In Weka software, cross-validation of K-fold method is compared with J48 decision tree and random forest but still there is problem arising in predicting the accurate result.

For predicting the corona virus outbreak, ML-based prediction system has been proposed and the forecasting is achieved, results are proved [12]. To predict the death rate and for confirming the cases the LR and LASSO techniques are used. The main focus of deep learning techniques in this pandemic situation is to real time monitoring. To prevent virus spread and limit the clinical resources in the rapid evaluation of COVID-19, the CXR is used to diagnosis and managing patient [13]. Patch-based framework with neural network train the data more stable and mapped to local neural network for finding the radiation which is compared with the trained and proposed SOTA method. 
Dual-KA was developed [14] by correlating the two kidneys with multiple feature approach in the human body is to stimulate and predict the kidney function. In each iteration of multiple feature approach, the GHR is checked in the dual-KA. The proposed method is used for many problems like benchmark classification, prediction of time series and water in real world. Detection of cancer is also tested by the proposed method with flexibility in detecting and tracking the symptoms of corona virus in low cost. Using headset, the respiratory problems are detected which is simple in configuration [15]. The principle behind the detecting respiratory problem with simple configuration can be extended to predict heart rate, temperature and oxygen saturation with the help of sensors which can be one of the solutions in predicting the symptoms and detection of corona virus.

The structure of kidney with renal changes is correlated in dynamic time series [16] and the images are registered. It should be developed as a tool for measuring the arteriosclerosis. In biopsy, the arteriosclerosis is detected by correlating the deformed images, normalized volume with pressure gradients. To learn the specific features of CXR, the diverted data distribution is used which is modality specific transfer by collecting CXR in large-scale [17]. In CXR classification, the proposed model is compared to pertained weights of ImageNet. Further, the model is improved and fine-tuned while transferring the images during classification.

On the spatial neural network, the kidney is detected with 3D ultrasound images by changing the image shape to volume [18]. To increase the accuracy in detecting the kidney function, the modelling of shape and classification of voxel was introduced and it validates texshape-based method superiority. To monitor the protein leak in urine, the POCT model was developed which is reliable. It is designed and developed with low cost economically portable. The protein leak is based on the total protein concentrate and creatinine in urine. It is color intensity change method with sensors. The detection of kidney disease is tool kit [19] which monitors the creatinine, protein concentrate of urine and display immediately which helps to cure in early stages. The model is designed in such a way that is user friendly which monitors and display amount of protein leaked in urine.

The ventilator is highly required for corona patients in case of severe where the capacity of airflow is expanded. So, the smart system was developed where the ventilator can be separated and used for two patients at a time [20]. The shortage of ventilator is raised as major problem in this pandemic situation. To avoid this problem the parallel model that supports the ventilator shortages was developed. The VSRS system was designed with user interface to avoid the potential error with effort. The hybrid CNN is used in internet of medical platform to predict the kidney disease [21] was developed to detect the small lesions in the renal.

To address the corona virus detection and prediction using deep learning methods there is war between the contents in online. The LDA algorithm [22] is used to collect the related information from the available sources regarding vaccines and development. To handle the large dataset the statistical methods are developed which are potential in labelling the disease. To classify the CT images, the weakly supervised deep learning [23] was developed to reduce the time delay of decision taking and treatment for the kidney is recommended. The deep learning framework is designed which extracts the CT images from different sources which includes scanners and centers. It is faster than established model and automatically classifies the images accurately. It helps the patients 
by providing warning messages regarding the severity of the disease and treatment. The crossbar-net [24] is the segmentation model mainly focused on size and of patches. Various image patches are trained in cascaded network to learn about the structure of renal with and without abnormality. This achieves the fast, accurate diagnosis and treatment of various kidney tumors with varying size and shape.

\section{Proposed Framework}

The solution for COVID-19 is a challenging task. The experiments on vaccines are still going on but the effects related to corona virus infection are increasing day by day. Early stage of corona virus caused infection only in respiratory organ and heart. But other organs are affected which is found later once the corona infection is cured. Many applications and wearable gadgets are introduced to detect the corona virus manually in home. Also, the smart devices are invented to detect the damage or infection in the lungs and heart due to COVID-19. Other than lungs many organs are affected which is not considered as conversational cue. Researchers found that it affects the kidney, pancreas and intestine in various ways. The proposed work is about the detection of kidney protein leak, microscopic blood leak, Acute Pancreatitis (AP), gastrointestinal bleeding and post infectious dysmotility. Deep learning techniques have the ability to predict the disease

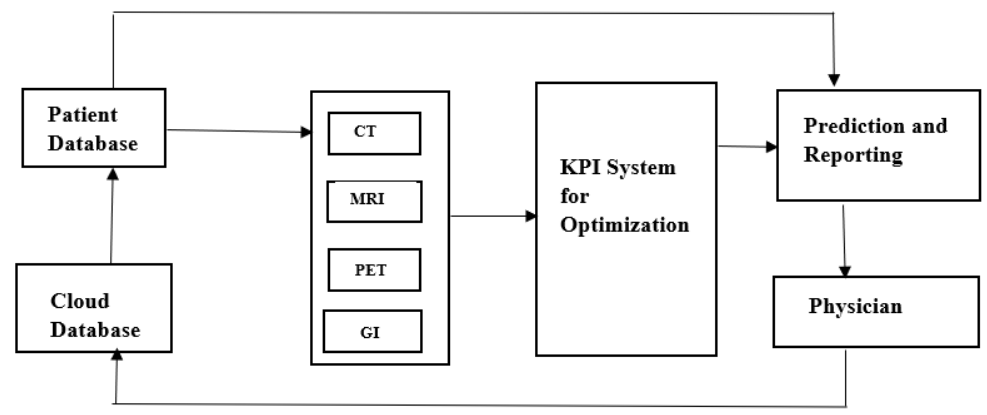

Figure 1. Architecture of KPI System

rapidly with the help of large dataset. It classifies the labels of disease which is helpful in diagnosing and treatment in the early stage. Deep learning works based on the neural networks; prediction is accurate if the network is deeper. It takes the input data without defining features and predicts the output more accurately. Various deep learning algorithm are used to predict the health diagnostics, here the Convolutional Neural Network (CNN) is used to predict the kidney, pancreas and intestine infection with the help of large data. Cloud computing provides security to the data which are highly confidential. Cloud plays a vital role in the healthcare system. After the prediction of health, the data are stored in the cloud securely for future use. Two databases are maintained namely patient database and cloud database as shown in Figure 1. Both the database holds the patient details and reports generated by the imaging system. Only the authorized user accesses the database to update, replace and delete the patient records. Imaging system holds the various scan reports which includes MRI, CT, PET and GI reports. Magnetic Resource Imaging (MRI) is used to take pictures of various tissues of pancreas, Com- 
puted Tomography (CT) is usually for diagnose disease or injury in the kidney, Positron Emission Tomography (PET) is for detecting cancer in the pancreas and Gastrointestinal Test (GI) is to detect the infection or injury in the small intestine and large intestine. These reports are used to optimize and improve the output of imaging system. The scan reports are used for tumor detection, chronic kidney disease and infections. Deep learning methods has the potential to detect the lesions in the kidney as in training phase, the CT images are trained with varying size and shape. It helps to learn the structure and function of kidneys and suggest treatment to physician which reduce the time, man power and cost. AI-based methods are incorporated in existing deep learning methods to overcome the outbreak of pandemic. Many kidney problems have no symptoms in early stage in such cases AI-based method helps to understand the function and structure of abnormalities in organs. This reduces the death rate and increases the life span of patients by detecting the disease in the early stage. The patient database holds the COVID-19 results and scan reports. The $\mathrm{CT}$ is combination of both X-rays and computer technology to take images horizontal or axial. CT scan of kidney is for prediction of tumors, chronic kidney disease and kidney stones. It detects the amount of protein and microscopic blood leak in urine. MRI is for confirming diagnosis and complications due to acute pancreatitis. PET measures the blood flow, oxygen use and functioning of pancreas. GI is for detecting the gastrointestinal bleeding and post infectious dysmotility. After fetching the scan reports it is optimized to improve the output of images to predict the affected parts using CNN. Images are improved and optimized by the CNN. Once the input images

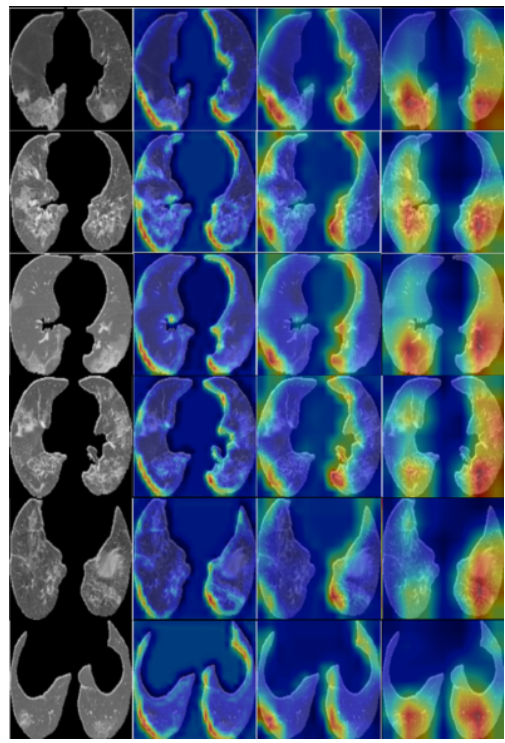

Figure 2. Optimization Results of CT images

are loaded in the $\mathrm{CNN}$, it generates its own feature by extracting from the input images. The feature is extracted to predict the health diagnostics as shown in Figure 2. The CNN is one of the top deep learning technologies which predict the output rapidly and accurate compared to other technique. The input image file is not directly taken by the CNN, rather than it converts the images into pixel values and passed to the input layer. First the 
input image pixel values are feature mapped by preserving the important features, then max pooling is applied in order to recognize the image and to predict the output as the input files vary in size and shape. Finally, the input values are given to the input layer, it undergoes through the layers to predict the output. Then it calculates the error rate in the output layer to check whether the predicted output matches the actual output. Again, it calculates the error rate by adjusting the weights. It processes until the CNN predicts the accurate output. Once the output is predicted, the report is generated whether the COVID-19 patients is affected with kidney infection, acute pancreatitis, gastrointestinal bleeding and post infectious dysmotility. Once the report is generated it is directed to the physician for cross checking and for treatment. The reports are stored in the cloud database for further use. The reports are accessed by the patients and physician to further treatment. It can be restored in the same cloud after the diagnosis which helps the other patients to predict the affected parts due to COVID-19. The proposed method is compared with other existing methods as shown in Table 1. The accuracy, sensitivity, specificity and F1 score are calculated it shows the proposed CNN methods achieves maximum accuracy than Support Vector Machine (SVM), Decision Tree, K-nearest neighbor $(\mathrm{KNN})$ and Random Forest (RF).

Table 1. Comparison of Performance between proposed and other methods

\begin{tabular}{|l|c|c|c|c|}
\hline Classifier & Accuracy & Sensitivity & Specificity & F1 score \\
\hline SVM & 85.79 & 86.00 & 86.56 & - \\
\hline Decision Tree & 87.98 & 87.88 & 86.23 & 87.80 \\
\hline KNN & 90.02 & 93.20 & 95.14 & 90.56 \\
\hline Random Forest & 97.36 & 98.98 & 98.74 & 97.43 \\
\hline Proposed Method & 99.86 & 100 & 99.80 & 99.78 \\
\hline
\end{tabular}

\section{Discussion}

Targeting on the feasibility of the CNN application for diagnosing COVID-19 related problems in organs, such as lungs, heart, kidney, liver, pancreas and intestine, CNN and cloud are used to suggest several DL-based methods. Other problems like high-risk patients, preventing from outbreak are recognized by DL methods. Advanced Deep Learning algorithms are integrated and analyzed the large data related to corona patients to understand the structure, function of virus, improving the speed and accuracy of detecting the organ dysfunction. It has been criticized that only some users are collecting the data, storing, managing and accessing. To avoid this, cloud computing is proposed in order to collect the huge corona patient data and maintained securely. It is used to predict and analyze the data more efficiently. However, AI-based methods are used to diagnosis the health condition because it monitors and collects data from several sources [25]. Various information regarding the COVID-19 from the articles and publications are concealed by suggested methods. While variety of inputs available publicly, clinical health data are shared as input for almost all AI and DL techniques. When people are surrounded by group they are characterized to be in high risk, so it is important to monitor the patients throughout the disease life period. The proposed method focused on kidney, pancreas and intestine of corona patients. These ideas are also extended to other high-risk 
patients because DL techniques are accurate in estimating the data and prediction. It is important that COVID-19 is spreading rapidly and affects the organs in second week of infection; therefore, the patients cannot be guarantee if they are recovered from the infection [25]. This clearly shows the importance of integrating the effective CNN-based method in understanding and detecting COVID-19. When data is given to this technique, it creates new data and feature as the training data. However, there are many techniques are available which predict and detect the disease by reducing the dependency in input. Though the proposed method has not been utilized earlier in case of diagnosing kidney, pancreas and intestine for effectiveness, there are several reports and source proved that speed, accuracy and efficiency is achieved by this method for similar kind of diseases. It is generalized that these methods are important for prediction in case of corona virus.

\section{Conclusion}

In this paper, based on ML and Cloud-based techniques with introduced concepts and platforms are proposed to deal with COVID-19. Incorporating COVID-19 diagnostic system such as RNN and CNN, many techniques have been developed. The main issues with COVID-19 like high-risk, geographical issues and recognizing have been discussed and studied in this work. The number of clinical and non-clinical datasets are used for prediction have been showed along with their mechanism for selecting models. By considering these platforms, it helps the experts and physician to analyze huge dataset, train machine, algorithms or optimize the analyzed data with more speed and accuracy. We discussed that they are suitable for creating workspace while experts and physician to work side by side. For this level of complexity, novel approaches have to be developed and real experiments should be conducted because advantages and limitations of ML methods for COVID-19 is yet to be achieved. The tools that developed to achieve sought goals and realize saving more lives from death.

\section{References}

[1] Chamola V, Hassija V, Gupta V, Guizani M. A comprehensive review of the COVID-19 pandemic and the role of IoT, drones, AI, blockchain, and 5G in managing its impact. Ieee access. 2020 May 4;8:9022565.

[2] Bhaskar N, Manikandan S. A deep-learning-based system for automated sensing of chronic kidney disease. IEEE Sensors Letters. 2019 Sep 18;3(10):1-4.

[3] Qin J, Chen L, Liu Y, Liu C, Feng C, Chen B. A machine learning methodology for diagnosing chronic kidney disease. IEEE Access. 2019 Dec 30;39(8):20991-1002.

[4] Xiao Z, Feng LN. A Study on Wireless Capsule Endoscopy for Small Intestinal Lesions Detection Based on Deep Learning Target Detection. IEEE Access. 2020 Aug 27;8:159017-26.

[5] Neumann K, Steil JJ. Optimizing extreme learning machines via ridge regression and batch intrinsic plasticity. Neurocomputing. 2013 Feb 15;102:23-30.

[6] Khan B, Naseem R, Muhammad F, Abbas G, Kim S. An empirical evaluation of machine learning techniques for chronic kidney disease prophecy. IEEE Access. 2020 Mar 18;8:55012-22.

[7] Jamshidi M, Lalbakhsh A, Talla J, Peroutka Z, Hadjilooei F, Lalbakhsh P, Jamshidi M, La Spada L, Mirmozafari M, Dehghani M, Sabet A. Artificial intelligence and COVID-19: deep learning approaches for diagnosis and treatment. Ieee Access. 2020 Jun 12;8:109581-95.

[8] Zhang H, Chen Y, Song Y, Xiong Z, Yang Y, Wu QJ. Automatic kidney lesion detection for CT images using morphological cascade convolutional neural networks. IEEE Access. 2019 Jun 20;7:83001-11. 
[9] Mohammed MA, Abdulkareem KH, Al-Waisy AS, Mostafa SA, Al-Fahdawi S, Dinar AM, Alhakami W, Abdullah BA, Al-Mhiqani MN, Alhakami H, Arbaiy N. Benchmarking methodology for selection of optimal COVID-19 diagnostic model based on entropy and TOPSIS methods. Ieee Access. 2020 May 19;8:99115-31.

[10] Sobrinho A, Queiroz AC, Da Silva LD, Costa ED, Pinheiro ME, Perkusich A. Computer-aided diagnosis of chronic kidney disease in developing countries: A comparative analysis of machine learning techniques. IEEE Access. 2020 Feb 3;8:25407-19.

[11] Rustam F, Reshi AA, Mehmood A, Ullah S, On BW, Aslam W, Choi GS. COVID-19 future forecasting using supervised machine learning models. IEEE access. 2020 May 25;8:101489-99.

[12] Oh Y, Park S, Ye JC. Deep learning covid-19 features on cxr using limited training data sets. IEEE transactions on medical imaging. 2020 May 8;39(8):2688-700.

[13] Abdullah S, Jaddi NS. Dual kidney-inspired algorithm for water quality prediction and cancer detection. IEEE Access. 2020 Jun 11;8:109807-20.

[14] Stojanović R, Škraba A, Lutovac B. A headset like wearable device to track COVID-19 symptoms. In2020 9th Mediterranean Conference on Embedded Computing (MECO) 2020 Jun 8 (pp. 1-4). IEEE.

[15] Hodneland E, Keilegavlen E, Hanson EA, Andersen E, Monssen JA, Rørvik J, Leh S, Marti HP, Lundervold A, Svarstad E, Nordbotten JM. In vivo detection of chronic kidney disease using tissue deformation fields from dynamic MR imaging. IEEE Transactions on Biomedical Engineering. 2018 Nov 2;66(6):1779-90.

[16] Rajaraman S, Siegelman J, Alderson PO, Folio LS, Folio LR, Antani SK. Iteratively pruned deep learning ensembles for COVID-19 detection in chest X-rays. IEEE Access. 2020 Jun 19;8:115041-50.

[17] Marsousi M, Plataniotis KN, Stergiopoulos S. Kidney Detection in 3-D Ultrasound Imagery via Shapeto-Volume Registration Based on Spatially Aligned Neural Network. IEEE journal of biomedical and health informatics. 2018 Feb 13;23(1):227-42.

[18] Mandal N, Dutta S, Gupta A, Bandyopadhyay D. Based Sensors for Point-of-Care Kidney Function Monitoring. IEEE Sensors Journal. 2020 Apr 24;20(17):9644-51.

[19] Kaplan M, Kneifel C, Orlikowski V, Dorff J, Newton M, Howard A, Shinn D, Bishawi M, Chidyagwai S, Balogh P, Randles A. Cloud computing for covid-19: lessons learned from massively parallel models of ventilator splitting. Computing in Science \& Engineering. 2020 Sep 21;22(6):37-47.

[20] Chen G, Ding C, Li Y, Hu X, Li X, Ren L, Ding X, Tian P, Xue W. Prediction of chronic kidney disease using adaptive hybridized deep convolutional neural network on the internet of medical things platform. IEEE Access. 2020 May 18;8:100497-508.

[21] Sear RF, Velásquez N, Leahy R, Restrepo NJ, El Oud S, Gabriel N, Lupu Y, Johnson NF. Quantifying COVID-19 content in the online health opinion war using machine learning. Ieee Access. 2020 May 11;8:91886-93.

[22] Hu S, Gao Y, Niu Z, Jiang Y, Li L, Xiao X, Wang M, Fang EF, Menpes-Smith W, Xia J, Ye H. Weakly supervised deep learning for covid-19 infection detection and classification from ct images. IEEE Access. 2020 Jun 29;8:118869-83.

[23] Yu Q, Shi Y, Sun J, Gao Y, Zhu J, Dai Y. Crossbar-net: A novel convolutional neural network for kidney tumor segmentation in ct images. IEEE transactions on image processing. 2019 Mar 18;28(8):4060-74.

[24] McCall B. COVID-19 and artificial intelligence: protecting health-care workers and curbing the spread. The Lancet Digital Health. 2020 Apr 1;2(4):166-7.

[25] Greenhalgh T, Koh GC, Car J. Covid-19: a remote assessment in primary care. Journal of Biomedical. 2020 Mar 25;368:1-5. 